Prescribing and monitoring high dose and combined antipsychotics in community mental health team

Dipanjan Banerjee ${ }^{1 \star}$, Tracey Green ${ }^{2}$ and Umama Khan ${ }^{3}$

${ }^{1}$ East Sussex Healthcare NHS Trust; ${ }^{2}$ St Marys Hospital and ${ }^{3}$ St Marys Hospital

${ }^{\star}$ Corresponding author.

doi: 10.1192/bjo.2021.476

Aims. The aim was to establish the prevalence of prescription of combined and high dose antipsychotics in the community mental health team and to see if such patients were being offered regular monitoring as advised by NICE guidelines.

Background. The use of high dose antipsychotic treatment (HDAT) should be in line with the recommendations of the Royal College of Psychiatrists Consensus statement on high dose antipsychotic Treatment. Such treatment should be initiated only when standard treatments have failed. As high dose prescribing of antipsychotic medications can potentially harm than benefit causing serious side effects and sudden death. Close monitoring and documentation are required. Also, reviewing these patients at regular intervals is recommended.

Method. A retrospective audit of 50 case notes of patients currently on antipsychotics was done. Case notes were selected randomly from a pool of 300 plus patients under the care of Isle of Wight NHS Trust in the HoNos Cluster 11 and 12 care pathways. Data were gathered on patients' demographics, diagnosis, medication monitoring. Data were analysed and discussed with consultant psychiatrist and senior mental health pharmacist. Patients who were not on any antipsychotic medications were not included in the audit.

Result. $90 \%$ of the patients were on single antipsychotic (45 out of 50 ), $4.45 \%$ (2 out of 45 )were above BNF recommended dose. 10\% (5) patients were prescribed combined antipsychotics. $40 \%$ (2)of them were above BNF recommended maximum dose. A total of $8 \%$ ( 4 out of 50 ) patients were on above BNF recommended maximum dose. All the patients on high dose antipsychotics had a clear plan documented in the system. Documented monitoring of Full blood count was found in $75 \%$, blood glucose in $50 \%$, lipid in $75 \%$, cardiac monitoring (Electrocardiogram or ECG) in $0 \%$, physical health monitoring in $0 \%$

Conclusion. As far as we know, this is the first time an audit has been done on the patients under the Community Mental Health Team on high dose antipsychotic treatment. The data showed areas of good practice as the majority of the patients were on monotherapy, and all the patients on a high dose or combined antipsychotics had clear management plans outlined in their notes. However, the audit also highlighted areas that currently need improvements such as regular monitoring of the patients on high dose and combined antipsychotics. It should also be clearly documented, recorded, and reviewed at six-monthly intervals.

\section{Co-morbid gambling disorder in a local drug and alcohol service: an audit to determine prevalence}

John Barker ${ }^{1 \star}$, Ruta Rele ${ }^{1}$, Charlotte Cartwright ${ }^{2}$ and Bethany Dinsdale-Young ${ }^{3}$

${ }^{1}$ Sheffield Health and Social Care NHS Foundation Trust; ${ }^{2}$ The University of Sheffield and ${ }^{3}$ The University of Sheffield ${ }^{\star}$ Corresponding author.

\section{doi: 10.1192/bjo.2021.477}

Aims. National surveys show that over 56\% of adults in England gamble annually, and of those surveyed, $0.5 \%$ were problem gamblers, equating to 300,000 problem gamblers at any point. The prevalence of problem gambling in patients with a substance misuse disorder ranges from $20.5 \%$ to $55 \%$.

The audit aims to improve the care of patients with comorbid substance misuse and gambling disorder by assessing the extent to which the service currently enquires about and records problem gambling in its patient cohort.

It is hypothesised that as no formal recording process is in place locally, this information will not be recorded systematically and in a way that is easily retrievable by the service.

The audit will allow the service to assess whether changes need to be made to the initial assessment pathways into treatment for substance-related disorders to adequately record this information so that further assessment and onward referral can take place.

Method. All active patients $(n=2824)$ within the service had both their electronic initial assessments and their entire electronic notes screened for terms such as 'betting' and 'gambling' and this was recorded using an Excel spreadsheet. Prevalence rates across the teams (opiates, non-opiates and alcohol) were then calculated.

Result. The results showed that $0 \%$ of patients had any entries in their initial screening noting any gambling activity. Further scrutiny of the records revealed that only 3.5\% (n-99) had ever discussed gambling with a worker in any of the services.

Conclusion. The majority $(n=52)$ of patients who had discussed gambling only had one positive search result, suggesting this was not followed-up in a systematic fashion. Recommendations are to revise the common assessment pro-forma to include a validated brief screening tool (lie/bet), where one positive answer triggers a further assessment with an appropriate clinician for consideration of referral to the local NHS gambling service.

Improving patient waiting times and quality of care by arranging access to notes from a neighbouring trust

Georgios Basdanis* and Cormac Fenton

Mental Health Liaison Team, St Thomas' Hospital, South London and Maudsley NHS Foundation Trust

${ }^{\star}$ Corresponding author.

doi: $10.1192 /$ bjo.2021.478

Aims. We aim to improve waiting times in the Emergency Department and improve the overall quality of care of out-of-area patients by arranging for the liaison team to have access to the electronic notes system of a neighbouring trust.

Method. St Thomas' Hospital is located in south London, right opposite the City of Westminster. As a result, approximately $20 \%$ of patients we see in mental health liaison are from that locality. Given that they belong to a different trust, we do not have access to their notes, which leads to a delay in trying to establish whether they are known to local mental health services. Often, staff are reluctant to divulge information. When information is shared, it is often late and/or incomplete. We approached the Chief Clinical Information Officer and Head of Information Governance from Central and North West London (CNWL) NHS Foundation Trust. We held weekly meetings which included both IT departments. Our IT had to install the electronic notes application (SystmOne) on our computers and open relevant firewall ports. The information is access through an NHS Smartcard, therefore CNWL had to authorise read-only Smartcard profiles for every member of the liaison team. A quick reference guide was created for all staff that would be using the new application. The system went live on 21 January 2021.

Result. We audited patient outcomes in December 2020 and February 2021 for initial comparison. In December 2020, the 